УДК $512.53+512.57$

\author{
Yu. V. ZHUCHOK
}

\title{
ENDOMORPHISMS OF FREE ABELIAN MONOGENIC DIGROUPS
}

Yu. V. Zhuchok. Endomorphisms of free abelian monogenic digroups, Mat. Stud. 43 (2015), $144-152$.

We construct a free abelian monogenic digroup and describe its endomorphism semigroup.

Ю. В. Жучок. Эндоморфизмы свободных абелевых моногенных дигрупп // Мат. Студії. 2015. - T.43, №2. - C.144-152.

Определяется конструкция свободной абелевой моногенной дигруппы и описывается ее полугруппа эндоморфизмов.

1. Introduction. The notion of a digroup first appeared in the work of Jean-Louis Loday ([1]). An algebraic system $(D, \dashv, \vdash)$ with two binary associative operations $\dashv$ and $\vdash$ is called a digroup if for all $x, y, z \in D$ the following conditions hold:

$\left(D_{1}\right)(x \dashv y) \dashv z=x \dashv(y \vdash z)$,

$\left(D_{2}\right)(x \vdash y) \dashv z=x \vdash(y \dashv z)$,

$\left(D_{3}\right)(x \dashv y) \vdash z=x \vdash(y \vdash z)$,

$\left(D_{4}\right)$ there exists $e \in D$ such that for all $x \in D, e \vdash x=x=x \dashv e$,

$\left(D_{5}\right)$ for all $x \in D$ there exists a unique element $x^{-1} \in D$ such that $x \vdash x^{-1}=e=x^{-1} \dashv x$.

An element $e$ is called a bar-unit of $(D, \dashv, \vdash)$ and $x^{-1}$ is said to be inverse to $x$ with respect to $e$. It should be noted that this definition does not imply that $e$ is the unique bar-unit of $D$. In general the digroup can have many bar-units. If operations of a digroup coincide, the digroup becomes a group. One of the first results about digroups is the proof of the fact that Cayley's theorem for groups has an analogue in the class of all digroups ([2]). M. K. Kinyon modified Loday's terminology to give a much cleaner definition of a digroup and then used semigroup theory to show that every digroup is the product of a group and a trivial digroup ([3]). An even simpler basis of independent axioms for the variety of digroups was obtained by J. D. Phillips ([4]). Some structural properties of digroups were studied in [5]. More information on digroups and their examples can be found, for example, in [6], [7].

It is well-known that the notion of a digroup is closely related with the notion of a dimonoid ([1]). Recall that a nonempty set $D$ equipped with two binary associative operations $\dashv$ and $\vdash$ satisfying the axioms $\left(D_{1}\right)-\left(D_{3}\right)$ is called a dimonoid. Dimonoids have been studied

2010 Mathematics Subject Classification: 08A05.

Keywords: free abelian digroup; dimonoid; congruence; endomorphism.

doi:10.15330/ms.43.2.144-152

(C) Yu. V. Zhuchok, 2015 
by many authors (see, e.g., [8]-[10]). Dimonoids and in particular digroups play a prominent role in problems from the theory of Leibniz algebras. In this paper we study a free abelian monogenic digroup and its endomorphism semigroup.

The paper is organized as follows. In Section 2, we give necessary definitions and construct a free abelian monogenic digroup. In Section 3, we define the least congruence on a free dimonoid such that the corresponding quotient is isomorphic to the free abelian monogenic digroup. In Section 4, we describe all endomorphisms of the free abelian monogenic digroup and construct a semigroup which is isomorphic to the endomorphism monoid of the given free digroup.

2. The free abelian monogenic digroup. A digroup $(D, \dashv, \vdash)$ is called abelian if $x \dashv y=$ $y \vdash x$ for all $x, y \in D[6]$. A digroup generated by one element is called monogenic.

Let $G$ be an arbitrary abelian additive group, $X_{1}, X_{2}, \ldots, X_{n-1}$ be non-empty subsets of $G$ and $X_{n}=G(n \geq 2)$. We denote by $\prod_{i=1}^{n} X_{i}$ the direct product $X_{1} \times X_{2} \times \ldots \times X_{n}$ and set $x^{+}=x_{1}+x_{2}+\ldots+x_{n}$ for all $x=\left(x_{1}, x_{2}, \ldots, x_{n}\right) \in \prod_{i=1}^{n} X_{i}$.

We take arbitrary $x, y \in \prod_{i=1}^{n} X_{i}$ and define two binary operations $\dashv$ and $\vdash$ on $\prod_{i=1}^{n} X_{i}$ as follows: $x \dashv y=\left(x_{1}, x_{2}, \ldots, x_{n-1}, x_{n}+y^{+}\right), \quad x \vdash y=\left(y_{1}, y_{2}, \ldots, y_{n-1}, y_{n}+x^{+}\right)$.

Proposition 1. The algebraic system $\left(\prod_{i=1}^{n} X_{i}, \dashv, \vdash\right)$ is an abelian digroup.

Proof. Let $x, y, z \in \prod_{i=1}^{n} X_{i}$. Then

$$
\begin{aligned}
(x \dashv y) \dashv z= & \left(x_{1}, \ldots, x_{n-1}, x_{n}+y^{+}\right) \dashv\left(z_{1}, z_{2}, \ldots, z_{n}\right)=\left(x_{1}, \ldots, x_{n-1}, x_{n}+y^{+}+z^{+}\right)= \\
& =\left(x_{1}, x_{2} \ldots, x_{n}\right) \dashv\left(y_{1}, \ldots, y_{n-1}, y_{n}+z^{+}\right)=x \dashv(y \dashv z), \\
(x \vdash y) \vdash z= & \left(y_{1}, \ldots, y_{n-1}, y_{n}+x^{+}\right) \vdash\left(z_{1}, z_{2}, \ldots, z_{n}\right)=\left(z_{1}, \ldots, z_{n-1}, z_{n}+y^{+}+x^{+}\right)= \\
& =\left(x_{1}, x_{2} \ldots, x_{n}\right) \vdash\left(z_{1}, \ldots, z_{n-1}, z_{n}+y^{+}\right)=x \vdash(y \vdash z) .
\end{aligned}
$$

Thus, operations $\dashv$ and $\vdash$ are associative. Show that axioms $\left(D_{1}\right)-\left(D_{3}\right)$ hold:

$$
\begin{gathered}
(x \dashv y) \dashv z=\left(x_{1}, \ldots, x_{n-1}, x_{n}+y^{+}+z^{+}\right)= \\
=\left(x_{1}, x_{2} \ldots, x_{n}\right) \dashv\left(z_{1}, \ldots, z_{n-1}, z_{n}+y^{+}\right)=x \dashv(y \vdash z), \\
(x \vdash y) \dashv z=\left(y_{1}, \ldots, y_{n-1}, y_{n}+x^{+}\right) \dashv\left(z_{1}, z_{2}, \ldots, z_{n}\right)=\left(y_{1}, \ldots, y_{n-1}, y_{n}+x^{+}+z^{+}\right)= \\
=\left(x_{1}, x_{2} \ldots, x_{n}\right) \vdash\left(y_{1}, \ldots, y_{n-1}, y_{n}+z^{+}\right)=x \vdash(y \dashv z), \\
(x \dashv y) \vdash z=\left(x_{1}, \ldots, x_{n-1}, x_{n}+y^{+}\right) \vdash\left(z_{1}, z_{2}, \ldots, z_{n}\right)= \\
=\left(z_{1}, \ldots, z_{n-1}, z_{n}+y^{+}+x^{+}\right)=x \vdash(y \vdash z) .
\end{gathered}
$$

Therefore, $\left(\prod_{i=1}^{n} X_{i}, \dashv, \vdash\right)$ is a dimonoid.

Let $e$ be an arbitrary bar-unit of $\left(\prod_{i=1}^{n} X_{i}, \dashv, \vdash\right)$. Then for all $x \in \prod_{i=1}^{n} X_{i}$ we obtain

$$
e \vdash x=\left(x_{1}, \ldots, x_{n-1}, x_{n}+e^{+}\right)=\left(x_{1}, x_{2}, \ldots, x_{n}\right)=x \dashv e .
$$

It follows that $e^{+}=0$. It is clear, if $e \in \prod_{i=1}^{n} X_{i}$ such that $e^{+}=0$, then $e$ is a bar-unit of $\left(\prod_{i=1}^{n} X_{i}, \dashv, \vdash\right)$.

Fix a bar-unit $e$ of $\left(\prod_{i=1}^{n} X_{i}, \dashv, \vdash\right)$ and assume that for some $x \in \prod_{i=1}^{n} X_{i}$ there exists $x^{-1}=\left(y_{1}, y_{2}, \ldots, y_{n}\right) \in \prod_{i=1}^{n} X_{i}$ such that $x \vdash x^{-1}=\left(y_{1}, \ldots, y_{n-1}, y_{n}+x^{+}\right)=\left(e_{1}, e_{2}, \ldots, e_{n}\right)=$ $x^{-1} \dashv x$.

Hence $x^{-1}=\left(e_{1}, \ldots, e_{n-1}, e_{n}-x^{+}\right)$. Besides, $x^{-1}$ is a unique inverse element to $x$ with respect to $e$. So, $\left(\prod_{i=1}^{n} X_{i}, \dashv, \vdash\right)$ is a digroup.

Finally, we have $x \dashv y=\left(x_{1}, \ldots, x_{n-1}, x_{n}+y^{+}\right)=y \vdash x$ for all $x, y \in \prod_{i=1}^{n} X_{i}$. 
Let $\mathbb{N}$ be the set of all natural numbers, $\mathbb{N}^{0}=\mathbb{N} \cup\{0\}$ and $E=\{1,-1\}$. Denote by $(\mathbb{Z},+)$ the additive group of all integer numbers.

By Proposition 1, the algebraic sysytem $(E \times \mathbb{Z},-\dashv, \vdash)$ is an abelian digroup. Bar-units of $(E \times \mathbb{Z}, \dashv, \vdash)$ are $(1,-1)$ and $(-1,1)$.

For every element $x$ of an arbitrary digroup $(D, \dashv, \vdash)$ we use denotations:

$$
x_{\vdash}^{n}=\underbrace{x \vdash x \vdash \ldots \vdash x}_{n}, x_{\dashv}^{n}=\underbrace{x \dashv x \dashv \ldots \dashv x}_{n}(n \in \mathbb{N}) .
$$

Lemma 1. Each of sets $\{(1,0)\},\{(-1,0)\}$ is generating for the digroup $(E \times \mathbb{Z}, \dashv, \vdash)$.

Proof. Show that $\{(1,0)\}$ is a generating set of $(E \times \mathbb{Z}, \dashv, \vdash)$. We take the bar-unit $(-1,1)$ as the acting of a nullary operation on $(E \times \mathbb{Z},-1, \vdash)$. Note that $(-1,0)$ is inverse to $(1,0)$ with respect to $(-1,1)$. It is not hard to check by an induction that for all $n \in \mathbb{N}^{0}$,

$$
(1,0)_{\vdash}^{n+1}=(1, n)=(1,0)_{\dashv}^{n+1}, \quad(-1,0)_{\vdash}^{n+1}=(-1,-n)=(-1,0)_{\dashv}^{n+1} .
$$

Then for all $n \in \mathbb{N}^{0}$ we obtain $(1,0) \dashv(-1,-n)=(1,-1-n), \quad(-1,0) \dashv(1, n)=$ $(-1,1+n)$.

Therefore, $\langle(1,0)\rangle=E \times \mathbb{Z}$. Analogously we can prove that $\{(-1,0)\}$ is a generating set of the digroup $(E \times \mathbb{Z}, \dashv, \vdash)$.

From this lemma immediately follows

Corollary 1. Let $(i, 0)_{\dashv}^{0}$ be the fixed bar-unit of $(E \times \mathbb{Z}, \dashv, \vdash)$ for all $i \in E$. Each element $(a, m)$ of $(E \times \mathbb{Z}, \dashv, \vdash)$ can be uniquely represented as $(a, m)=(a, 0) \dashv(i, 0)_{\dashv}^{m}$ for suitable $i \in E$.

It is not hard to check that for every element $x$ of an abelian digroup $(D,-1, \vdash)$ we have $x_{\vdash}^{n}=x_{\dashv}^{n}$ for all $n \in \mathbb{N}$. Therefore, for abelian digroups we write $x^{n}$ instead of $x_{\vdash}^{n}$.

Remark 1. For abelian digroups the identity $x^{m} \dashv x^{n}=x^{m+n}$ is not true for integers $m, n$. In order to satisfy this identity, it is enough that both its sides would have one common multiplier on the left (on the right) with respect to an operation $\dashv$ (respect. $\vdash$ ).

For example, take the digroup $(E \times \mathbb{Z},-\dashv, \vdash), x=(1,0) \in E \times \mathbb{Z}$ and $m=2, n=-4$. Then with respect to the bar-unit $(-1,1)$ we have $x^{m+n}=(1,0)^{-2}=(-1,-1) \neq(1,-3)=$ $(1,0)^{2} \dashv(1,0)^{-4}=x^{m} \dashv x^{n}$, however for all $(a, b) \in E \times \mathbb{Z},(a, b) \dashv x^{m+n}=(a, b-2)=$ $(a, b) \dashv x^{m} \dashv x^{n}$.

For arbitrary digroups $\mathfrak{D}_{1}=\left(D_{1}, \dashv_{1}, \vdash_{1}\right)$ and $\mathfrak{D}_{2}=\left(D_{2}, \dashv_{2}, \vdash_{2}\right)$, a mapping $\varphi: D_{1} \rightarrow D_{2}$ is called a homomorphism of $\mathfrak{D}_{1}$ into $\mathfrak{D}_{2}$ if for all $x, y \in D_{1}$ we have $\left(x \dashv_{1} y\right) \varphi=x \varphi \dashv_{2}$ $y \varphi, \quad\left(x \vdash_{1} y\right) \varphi=x \varphi \vdash_{2} y \varphi$.

A bijective homomorphism $\varphi: D_{1} \rightarrow D_{2}$ is called an isomorphism of $\mathfrak{D}_{1}$ into $\mathfrak{D}_{2}$. In this case digroups $\mathfrak{D}_{1}$ and $\mathfrak{D}_{2}$ are called isomorphic.

Theorem 1. The digroup $(E \times \mathbb{Z}, \dashv, \vdash)$ is a free abelian monogenic digroup.

Proof. Let $\left(D^{\prime}, \dashv^{\prime}, \vdash^{\prime}\right)$ be an arbitrary abelian digroup, $(1,0) \xi=t \in D^{\prime}$, and $(-1,0) \xi=t^{-1}$, where $t^{-1}$ is inverse to $t$ with respect to the fixed bar-unit $e^{\prime} \in D^{\prime}$. Further, we naturally extend $\xi$ to a mapping $\Xi$ of $E \times \mathbb{Z}$ into $D^{\prime}$ using the fact that $\{(1,0)\}$ is the generating set of 
$(E \times \mathbb{Z}, \dashv, \vdash)$ and $(1,0)^{-1}=(-1,0)$ (see Lemma 1), that is, $(a, m) \Xi=\left((a, 0) \dashv(i, 0)^{m}\right) \Xi=$ $t^{a} \dashv^{\prime} t^{i|m|}=t^{a} \dashv^{\prime} t^{m}$ for all $(a, m) \in E \times \mathbb{Z}$.

Assume that $(a, m),(b, n) \in E \times \mathbb{Z}$. Taking into account Remark 1 ,

$$
\begin{gathered}
((a, m) \dashv(b, n)) \Xi=(a, m+b+n) \Xi=t^{a} \dashv^{\prime} t^{m+b+n}=t^{a} \dashv^{\prime}\left(t^{m} \dashv^{\prime} t^{b} \dashv^{\prime} t^{n}\right)= \\
=\left(t^{a} \dashv^{\prime} t^{m}\right) \dashv^{\prime}\left(t^{b} \dashv^{\prime} t^{n}\right)=(a, m) \Xi \dashv^{\prime}(b, n) \Xi .
\end{gathered}
$$

By Proposition 1, the digroup $(E \times \mathbb{Z}, \dashv, \vdash)$ is abelian. Since $\left(D^{\prime}, \dashv^{\prime}, \vdash^{\prime}\right)$ is an abelian digroup also, we obtain

$$
((a, m) \vdash(b, n)) \Xi=((b, n) \dashv(a, m)) \Xi=(b, n) \Xi \dashv^{\prime}(a, m) \Xi=(a, m) \Xi \vdash^{\prime}(b, n) \Xi .
$$

Thus, $\Xi$ is a homomorphism of $(E \times \mathbb{Z}, \dashv, \vdash)$ into $\left(D^{\prime}, \dashv^{\prime}, \vdash^{\prime}\right)$. In addition, $(E \times \mathbb{Z}) \Xi$ is generated by one element $t$.

3. The least abelian digroup congruence. Let $(D, \dashv, \vdash)$ be an arbitrary dimonoid, $\rho$ be an equivalence relation on $D$ which is stable on the left and on the right with respect to each of operations $\dashv, \vdash$. In this case $\rho$ is called a congruence on $(D, \dashv, \vdash)$.

For a congruence $\rho$ on a dimonoid $(D, \dashv, \vdash)$ the corresponding quotient-dimonoid is denoted by $(D,-\dashv, \vdash) / \rho$. A congruence $\rho$ on a dimonoid $(D, \dashv, \vdash)$ is called abelian digroup if the quotient-dimonoid $(D, \dashv, \vdash) / \rho$ is an abelian digroup.

Now we define a free dimonoid on an arbitrary set $Y$. Put $\widetilde{Y}=\{\widetilde{y} \mid y \in Y\}$. Two binary operations $\dashv$ and $\vdash$ are defined on the set

$$
\operatorname{Fd}(Y)=\tilde{Y} \cup(\tilde{Y} \times Y) \cup(Y \times \tilde{Y}) \cup(\tilde{Y} \times Y \times Y) \cup(Y \times \tilde{Y} \times Y) \cup(Y \times Y \times \tilde{Y}) \cup \ldots
$$

as follows:

$$
\begin{aligned}
& \left(y_{1}, \ldots, \widetilde{y}_{i}, \ldots, y_{k}\right) \prec\left(y_{k+1}, \ldots, \widetilde{y}_{j}, \ldots, y_{l}\right)=\left(y_{1}, \ldots, \widetilde{y}_{i}, \ldots, y_{l}\right), \\
& \left(y_{1}, \ldots, \widetilde{y}_{i}, \ldots, y_{k}\right) \succ\left(y_{k+1}, \ldots, \widetilde{y}_{j}, \ldots, y_{l}\right)=\left(y_{1}, \ldots, \widetilde{y}_{j}, \ldots, y_{l}\right) .
\end{aligned}
$$

The algebra $(\operatorname{Fd}(Y), \prec, \succ)$ is the free dimonoid (see [1]). Elements of $\operatorname{Fd}(Y)$ are called words and $\widetilde{Y}$ is the generating set of $(\operatorname{Fd}(Y), \prec, \succ)$.

Let $X=\left\{x, x^{-1}\right\}$ and $(\operatorname{Fd}(X), \prec, \succ)$ be the free dimonoid on $X$. By $q_{\hat{t}}(w), t \in X$, we denote the quantity of all letters $\widetilde{t}$ that are included in the canonical form of $w=$ $\left(w_{1}, \ldots, \widetilde{w_{k}}, \ldots, w_{l}\right): w=\widetilde{w_{1}} \succ \ldots \succ \widetilde{w_{k}} \prec \ldots \prec \widetilde{w_{l}}$.

For every $w \in \operatorname{Fd}(X)$ we put $q(w)=q_{\widetilde{x}}(w)-q_{\widetilde{x^{-1}}}(w)$. Define a binary relation $\sigma$ on $\operatorname{Fd}(X)$ as follows: words $u=\left(u_{1}, \ldots, \widetilde{u_{i}}, \ldots, u_{n}\right)$ and $v=\left(v_{1}, \ldots, \widetilde{v_{j}}, \ldots, v_{m}\right)$ of $\operatorname{Fd}(X)$ are $\sigma$-equivalent if $u_{i}=v_{j}$ and $q(u)=q(v)$.

A word $u=\left(\widetilde{u_{1}}, \ldots, u_{i}, \ldots, u_{n}\right) \in \operatorname{Fd}(X)$ we call irreducible if it do not contain any subword of the form $\left(x, x^{-1}\right),\left(x^{-1}, x\right)$. For example, irreducible words of $\operatorname{Fd}(X)$ are $\widetilde{x}$, $(\widetilde{x}, x, x),\left(\widetilde{x}, x^{-1}, x^{-1}, x^{-1}\right)$ and $\widetilde{x^{-1}},\left(\widetilde{x^{-1}}, x^{-1}\right),\left(\widetilde{x^{-1}}, x, x, x, x\right)$.

Lemma 2. The relation $\sigma$ is a congruence on the free dimonoid $(\operatorname{Fd}(X), \prec, \succ)$ such that for any class $[w] \in(\operatorname{Fd}(X), \prec, \succ) / \sigma$ there exists a unique irreducible word $w^{\prime} \in[w]$ of the form $w^{\prime}=\widetilde{y} v, y \in X, v \in X^{*} \cup\left(X^{-1}\right)^{*}$, where $X^{*}$ and $\left(X^{-1}\right)^{*}$ are free monoids on $X$ and $X^{-1}$, respectively. 
Proof. It easy to see that $\sigma$ is an equivalence relation. Assume that $u=\left(u_{1}, \ldots, \widetilde{u}_{i}, \ldots, u_{n}\right), v=$ $\left(v_{1}, \ldots, \widetilde{v_{j}}, \ldots, v_{m}\right) \in \operatorname{Fd}(X)$ such that $u \sigma v$ and $w=\left(w_{1}, \ldots, \widetilde{w_{k}}, \ldots, w_{l}\right) \in \operatorname{Fd}(X)$. Then

$$
\begin{aligned}
& u \prec w=\left(u_{1}, \ldots, \widetilde{u}_{i}, \ldots, u_{n}, w_{1}, \ldots, w_{l}\right), v \prec w=\left(v_{1}, \ldots, \widetilde{v_{j}}, \ldots, v_{m}, w_{1}, \ldots, w_{l}\right), \\
& u \succ w=\left(u_{1}, \ldots, u_{n}, w_{1}, \ldots, \widetilde{w_{k}}, \ldots, w_{l}\right), \quad v \succ w=\left(v_{1}, \ldots, v_{m}, w_{1}, \ldots, \widetilde{w_{k}}, \ldots, w_{l}\right) .
\end{aligned}
$$

Since $u_{i}=v_{j}$ and $q(u \prec w)=q(v \prec w), q(u \succ w)=q(v \succ w)$, we have $(u \prec w) \sigma(v \prec w)$ and $(u \succ w) \sigma(v \succ w)$. Analogously we can show that $(w \prec u) \sigma(w \prec v)$ and $(w \succ u) \sigma$ $(w \succ v)$. Thus, $\sigma$ is a congruence.

Let $[w] \in(\operatorname{Fd}(X), \prec, \succ) / \sigma$ be an arbitrary congruence class, $w=\left(w_{1}, \ldots, \widetilde{w_{k}}, \ldots, w_{l}\right)$. By the definition of $\sigma$, such words as $w^{\prime \prime}=\left(\widetilde{w_{k}}, w_{1}, \ldots, w_{k-1}, w_{k+1}, \ldots, w_{l}\right)$ and

$$
\begin{aligned}
& \left(\widetilde{w_{k}}, x, x^{-1}, w_{1}, \ldots, w_{k-1}, w_{k+1}, \ldots, w_{l}\right), \ldots,\left(\widetilde{w_{k}}, w_{1}, \ldots, w_{k-1}, w_{k+1}, \ldots, w_{l}, x, x^{-1}\right), \\
& \left(\widetilde{w_{k}}, x^{-1}, x, w_{1}, \ldots, w_{k-1}, w_{k+1}, \ldots, w_{l}\right), \ldots,\left(\widetilde{w_{k}}, w_{1}, \ldots, w_{k-1}, w_{k+1}, \ldots, w_{l}, x^{-1}, x\right)
\end{aligned}
$$

are $\sigma$-equivalent to $w$. Thus, deleting from $w^{\prime \prime}$ all subwords of the form $\left(x, x^{-1}\right),\left(x^{-1}, x\right)$ (in the case if such subwords there exist), we obtain the irreducible word $w^{\prime}$ which is $\sigma$-equivalent to $w$. Uniqueness of $w^{\prime}$ is obvious, besides $w^{\prime}$ can be represented as $(\widetilde{x^{m}}, \underbrace{x^{n}, x^{n}, \ldots, x^{n}}_{s})$, where $m, n= \pm 1, s \geq 0$.

For convenient irreducible words of $\operatorname{Fd}(X)$ we will write as $\widetilde{x^{\alpha}} x^{\beta}$, where $\alpha \in E, \beta \in \mathbb{Z}$, in particular $\widetilde{x^{\alpha}} x^{0}=\widetilde{x^{\alpha}}$.

Lemma 3. The quotient-dimonoid $(\mathrm{Fd}(X), \prec, \succ) / \sigma$ is an abelian digroup isomorphic to the free digroup $(E \times \mathbb{Z}, \dashv, \vdash)$.

Proof. Lemma 2 implies $(\operatorname{Fd}(X), \prec, \succ) / \sigma$ is a dimonoid. Since $(u \dashv v) \sigma(v \vdash u)$ for all $u, v \in \operatorname{Fd}(X)$, we have $(\operatorname{Fd}(X), \prec, \succ) / \sigma$ is abelian. Let $[e] \in(\operatorname{Fd}(X), \prec, \succ) / \sigma$ such that $q_{\widetilde{x}}(e)=q_{x^{-1}}(e)$. Then for all $[w] \in(\operatorname{Fd}(X), \prec, \succ) / \sigma,[e] \succ[w]=[e \succ w]=[w]=[w] \prec[e]$.

Thus, $[e]$ is a bar-unit of $(\operatorname{Fd}(X), \prec, \succ) / \sigma$ for all $e \in \operatorname{Fd}(X)$ with $q(e)=0$. Moreover, for the fixed bar-unit $[e] \in(\operatorname{Fd}(X), \prec, \succ) / \sigma, e=\widetilde{x^{e_{1}}} x^{e_{2}}$, and $[w],[u] \in(\operatorname{Fd}(X), \prec, \succ) / \sigma$, where $w=\widetilde{x^{w_{1}}} x^{w_{2}}, u=\widetilde{x^{u_{1}}} x^{u_{2}}$, the equalities $\left.[w] \succ[u]=\widetilde{x^{w_{1}}} x^{w_{2}} \succ \widetilde{x^{u_{1}}} x^{u_{2}}\right]=\left[\widetilde{x^{u_{1}}} x^{w_{1}+w_{2}+u_{2}}\right]=$ $\left[\widetilde{x^{e_{1}}} x^{e_{2}}\right]=[u] \prec[w]$ imply $[u]=\left[\widetilde{x^{e_{1}}} x^{e_{2}-w_{1}-w_{2}}\right]=[w]^{-1}$. Uniqueness of $[w]^{-1}$ is obvious.

Now define a mapping $\varphi$ of $(\operatorname{Fd}(X), \prec, \succ) / \sigma$ into $(E \times \mathbb{Z},-\dashv, \vdash)$ by $[w] \varphi=\left(w_{1}, w_{2}\right)$ for all irreducible words $w=\widetilde{x^{w_{1}}} x^{w_{2}} \in \operatorname{Fd}(X)$. Taking into account Lemma 2, it is clear that $\varphi$ is a bijective mapping.

Further for all $[u],[v] \in(\operatorname{Fd}(X), \prec, \succ) / \sigma$, where $u=\widetilde{x^{u_{1}}} x^{u_{2}}, v=\widetilde{x^{v_{1}}} x^{v_{2}}$, we have

$$
\begin{gathered}
([u] \prec[v]) \varphi=\left[\widetilde{x^{u_{1}}} x^{u_{2}} \prec \widetilde{x^{v_{1}}} x^{v_{2}}\right] \varphi=\left[\widetilde{x^{u_{1}}} x^{u_{2}+v_{1}+v_{2}}\right] \varphi=\left(u_{1}, u_{2}+v_{1}+v_{2}\right)= \\
=\left(u_{1}, u_{2}\right) \dashv\left(v_{1}, v_{2}\right)=[u] \varphi \dashv[v] \varphi .
\end{gathered}
$$

Since digroups $(\operatorname{Fd}(X), \prec, \succ) / \sigma$ and $(E \times \mathbb{Z}, \dashv, \vdash)$ are abelian,

$$
(A \succ B) \varphi=(B \prec A) \varphi=B \varphi \dashv A \varphi=A \varphi \vdash B \varphi
$$

for all $A, B \in(\operatorname{Fd}(X), \prec, \succ) / \sigma$.

From this lemma it follows that for $(\operatorname{Fd}(X), \prec, \succ) / \sigma$ there exist only two distinct barunits $e_{1}=\left[\left(\widetilde{x}, x^{-1}\right)\right]$ and $\left.e_{2}=\left[\widetilde{\left(x^{-1}\right.}, x\right)\right]$. 
Theorem 2. The binary relation $\sigma$ is the least abelian digroup congruence on the free dimonoid $(\operatorname{Fd}(X), \prec, \succ)$ with $X=\left\{x, x^{-1}\right\}$.

Proof. The proof of this statement follows from Lemma 2 and Lemma 3.

4. Endomorphisms of the free abelian digroup of rank 1. For an arbitrary digroup $\mathfrak{D}=(D, \dashv, \vdash)$ by $\operatorname{End}(\mathfrak{D})$ we denote the endomorphism monoid of $\mathfrak{D}$. First, we describe all endomorphisms of the free abelian monogenic digroup.

Lemma 4. Let e be the fixed bar-unit of the free abelian digroup $(E \times \mathbb{Z},-1, \vdash)$ and $t \in E \times \mathbb{Z}$. A transformation $\xi_{e, t}$ of $(E \times \mathbb{Z}, \dashv, \vdash)$ defined by

$$
(a, n) \xi_{e, t}= \begin{cases}\left(t_{1}, n t^{+}+t_{2}\right), & \text { if } a=1, \\ \left(e_{1},(n-1) t^{+}+e_{2}\right), & \text { if } a=-1\end{cases}
$$

is an endomorphism.

Proof. For all $(a, n),\left(a^{\prime}, n^{\prime}\right) \in E \times \mathbb{Z}$, we have the following cases:

1) $a=a^{\prime}=1$, then

$$
\begin{gathered}
\left((1, n) \dashv\left(1, n^{\prime}\right)\right) \xi_{e, t}=\left(1, n+1+n^{\prime}\right) \xi_{e, t}=\left(t_{1},\left(n+1+n^{\prime}\right) t^{+}+t_{2}\right)= \\
=\left(t_{1}, n t^{+}+t_{2}\right) \dashv\left(t_{1}, n^{\prime} t^{+}+t_{2}\right)=(1, n) \xi_{e, t} \dashv\left(1, n^{\prime}\right) \xi_{e, t} ;
\end{gathered}
$$

2) $a=1, a^{\prime}=-1$, then

$$
\begin{gathered}
\left((1, n) \dashv\left(-1, n^{\prime}\right)\right) \xi_{e, t}=\left(1, n-1+n^{\prime}\right) \xi_{e, t}=\left(t_{1},\left(n-1+n^{\prime}\right) t^{+}+t_{2}\right)= \\
=\left(t_{1}, n t^{+}+t_{2}\right) \dashv\left(e_{1},\left(n^{\prime}-1\right) t^{+}+e_{2}\right)=(1, n) \xi_{e, t} \dashv\left(-1, n^{\prime}\right) \xi_{e, t} ;
\end{gathered}
$$

3) $a=-1, a^{\prime}=1$, then

$$
\begin{aligned}
& \left((-1, n) \dashv\left(1, n^{\prime}\right)\right) \xi_{e, t}=\left(-1, n+1+n^{\prime}\right) \xi_{e, t}=\left(e_{1},\left(n+n^{\prime}\right) t^{+}+e_{2}\right)= \\
& \quad=\left(e_{1},(n-1) t^{+}+e_{2}\right) \dashv\left(t_{1}, n^{\prime} t^{+}+t_{2}\right)=(-1, n) \xi_{e, t} \dashv\left(1, n^{\prime}\right) \xi_{e, t} ;
\end{aligned}
$$

4) $a=a^{\prime}=-1$, then

$$
\begin{aligned}
& \left((-1, n) \dashv\left(-1, n^{\prime}\right)\right) \xi_{e, t}=\left(-1, n-1+n^{\prime}\right) \xi_{e, t}=\left(e_{1},\left(n+n^{\prime}-2\right) t^{+}+e_{2}\right)= \\
& =\left(e_{1},(n-1) t^{+}+e_{2}\right) \dashv\left(e_{1},\left(n^{\prime}-1\right) t^{+}+e_{2}\right)=(-1, n) \xi_{e, t} \dashv\left(-1, n^{\prime}\right) \xi_{e, t} .
\end{aligned}
$$

From 1)-4) it follows that $\xi_{e, t} \in \operatorname{End}(E \times \mathbb{Z}, \dashv)$. Since $(E \times \mathbb{Z}, \dashv, \vdash)$ is an abelian digroup, $\xi_{e, t} \in \operatorname{End}(E \times \mathbb{Z}, \dashv, \vdash)$ for all $e, t \in E \times \mathbb{Z}, e^{2}=e$.

Note that endomorphisms $\xi_{e, t}, e, t \in E \times \mathbb{Z}$, where $e^{+}=0$, are not injective in general. For example, if $e=t, e^{2}=e$, we have $x \xi_{e, t}=e$ for all $x \in E \times \mathbb{Z}$.

Lemma 5. Let $x=\left(x_{1}, x_{2}\right) \in E \times \mathbb{Z}$ and $m \in \mathbb{N}$. Then $x^{m}=\left(x_{1}, x_{2}+(m-1) x^{+}\right)$.

Proof. The proof of this statement is obvious.

Lemma 6. Let $\xi$ be an arbitrary endomorphism of $(E \times \mathbb{Z},-\dashv, \vdash)$ and $(1,0) \xi=t$. Then $\xi=\xi_{e, t}$ for some bar-unit $e \in E \times \mathbb{Z}$. 
Proof. Assume that $(-1,1) \xi=e$. It is clear that $e^{2}=e$, i.e. $e$ is the bar-unit of $(E \times \mathbb{Z}, \dashv, \vdash)$. Then there exists a unique inverse element $t^{-1}=\left(e_{1}, e_{2}-t^{+}\right)$to $t$ with respect to $e$. By Corollary 1 , for all $(a, n) \in E \times \mathbb{Z}$ we have $(a, n)=(a, 0) \dashv(j, 0)^{n}$ for suitable $j \in E$. Using Lemma 5, we obtain the following cases:

1) $n \geq 0, a=1$, then $(1, n) \xi=\left((1,0)^{n+1}\right) \xi=t^{n+1}=\left(t_{1}, t_{2}+n t^{+}\right)$;

2) $-n<0, a=1$, then

$$
\begin{gathered}
(1,-n) \xi=\left((1,0) \dashv(-1,0)^{n}\right) \xi=t \dashv t^{-n}= \\
=\left(t_{1}, t_{2}\right) \dashv\left(e_{1}, e_{2}-t^{+}+(n-1)\left(e^{+}-t^{+}\right)\right)=\left(t_{1}, t_{2}-n t^{+}\right) ;
\end{gathered}
$$

3) $n \geq 0, a=-1$, then

$$
\begin{gathered}
(-1, n) \xi=\left((-1,0) \dashv(1,0)^{n}\right) \xi=t^{-1} \dashv t^{n}= \\
=\left(e_{1}, e_{2}-t^{+}\right) \dashv\left(t_{1}, t_{2}+(n-1) t^{+}\right)=\left(e_{1}, e_{2}+(n-1) t^{+}\right) ;
\end{gathered}
$$

4) $-n<0, a=-1$, then

$\left.(-1,-n) \xi=(-1,0)^{n+1}\right) \xi=\left(e_{1}, e_{2}-t^{+}\right)^{n+1}=\left(e_{1}, e_{2}-t^{+}+n\left(e^{+}-t^{+}\right)\right)=\left(e_{1}, e_{2}-(n+1) t^{+}\right)$.

From 1)-4) it follows that $\xi$ coincides with $\xi_{e, t}$ (see Lemma 4), where $e=(-1,1) \xi$.

Let $W$ be the set of all bar-units of $(E \times \mathbb{Z},-1, \vdash)$, that is $W=\{(1,-1),(-1,1)\}$. Consider a binary operation $\circ$ on $W \times(E \times \mathbb{Z})$ defined as follows

$$
(e, t) \circ(i, s)= \begin{cases}\left(\left(s_{1},-s_{1}\right),\left(s_{1}, t_{2} s^{+}+s_{2}\right)\right), & \text { if } e_{1}=t_{1}=1, \\ \left(\left(s_{1},-s_{1}\right),\left(i_{1}, t^{+} s^{+}-i_{1}\right)\right), & \text { if } e_{1}=1, t_{1}=-1, \\ \left(\left(i_{1},-i_{1}\right),\left(s_{1}, t_{2} s^{+}+s_{2}\right)\right), & \text { if } e_{1}=-1, t_{1}=1, \\ \left(\left(i_{1},-i_{1}\right),\left(i_{1}, t^{+} s^{+}-i_{1}\right)\right), & \text { if } e_{1}=t_{1}=-1 .\end{cases}
$$

It is clear that the operation $\circ$ is completed on $W \times(E \times \mathbb{Z})$.

Lemma 7. The algebra $(W \times(E \times \mathbb{Z}), \circ)$ is a monoid with the identity $((-1,1),(1,0))$.

Proof. Take arbitrary $(e, t),(i, s),(j, r) \in W \times(E \times \mathbb{Z})$ and put $A=((e, t) \circ(i, s)) \circ(j, r)$, $B=(e, t) \circ((i, s) \circ(j, r))$.

Assume that $e_{1}=t_{1}=i_{1}=s_{1}=1$. Then

$$
\begin{gathered}
A=\left(\left(s_{1},-s_{1}\right),\left(s_{1}, t_{2} s^{+}+s_{2}\right)\right) \circ(j, r)=\left(\left(r_{1},-r_{1}\right),\left(r_{1}, t_{2} s^{+} r^{+}+s_{2} r^{+}+r_{2}\right)\right)= \\
=\left(\left(r_{1},-r_{1}\right),\left(r_{1}, t_{2}\left(r^{+}+s_{2} r^{+}\right)+s_{2} r^{+}+r_{2}\right)\right)=(e, t) \circ\left(\left(r_{1},-r_{1}\right),\left(r_{1}, s_{2} r^{+}+r_{2}\right)\right)=B .
\end{gathered}
$$

For $e_{1}=t_{1}=i_{1}=s_{1}=-1$ we have

$$
\begin{gathered}
A=\left(\left(i_{1},-i_{1}\right),\left(i_{1}, t^{+} s^{+}-i_{1}\right)\right) \circ(j, r)= \\
=\left(\left(j_{1},-j_{1}\right),\left(j_{1}, t^{+} s^{+} r^{+}-j_{1}\right)\right)=(e, t) \circ\left(\left(j_{1},-j_{1}\right),\left(j_{1}, s^{+} r^{+}-j_{1}\right)\right)=B .
\end{gathered}
$$

Let $e_{1}=i_{1}=1, t_{1}=s_{1}=-1$. Then

$$
\begin{aligned}
A & =\left(\left(s_{1},-s_{1}\right),\left(i_{1}, t^{+} s^{+}-i_{1}\right)\right) \circ(j, r)=\left(\left(j_{1},-j_{1}\right),\left(r_{1}, r_{2}+\left(t^{+} s^{+}-i_{1}\right) r^{+}\right)\right)= \\
& =\left(\left(j_{1},-j_{1}\right),\left(r_{1}, t^{+} s^{+} r^{+}-r_{1}\right)\right)=(e, t) \circ\left(\left(r_{1},-r_{1}\right),\left(j_{1}, s^{+} r^{+}-j_{1}\right)\right)=B .
\end{aligned}
$$


If $e_{1}=i_{1}=-1, t_{1}=s_{1}=1$, then

$$
\begin{gathered}
A=\left(\left(i_{1},-i_{1}\right),\left(s_{1}, t_{2} s^{+}+s_{2}\right)\right) \circ(j, r)=\left(\left(j_{1},-j_{1}\right),\left(r_{1}, t_{2} s^{+} r^{+}+s_{2} r^{+}+r_{2}\right)\right)= \\
=\left(\left(j_{1},-j_{1}\right),\left(r_{1}, t_{2}\left(r^{+}+s_{2} r^{+}\right)+s_{2} r^{+}+r_{2}\right)\right)=(e, t) \circ\left(\left(j_{1},-j_{1}\right),\left(r_{1}, s_{2} r^{+}+r_{2}\right)\right)=B .
\end{gathered}
$$

In similar way all other cases are proved. Thus, $(W \times(E \times \mathbb{Z}), \circ)$ is a semigroup. A direct verification shows that an identity of $(W \times(E \times \mathbb{Z}), \circ)$ is $((-1,1),(1,0))$.

The main result of this paper is the following theorem.

Theorem 3. (i) For any $(e, t) \in W \times(E \times \mathbb{Z})$ a transformation $\xi_{e, t}$ of the free abelian monogenic digroup $(E \times \mathbb{Z}, \dashv, \vdash)$ defined by

$$
(a, n) \xi_{e, t}= \begin{cases}\left(t_{1}, n t^{+}+t_{2}\right), & \text { if } a=1, \\ \left(e_{1},(n-1) t^{+}+e_{2}\right), & \text { if } a=-1\end{cases}
$$

is an endomorphism. And every endomorphism of $(E \times \mathbb{Z}, \dashv, \vdash)$ has the above form.

(ii) The endomorphism monoid $\operatorname{End}(E \times \mathbb{Z}, \dashv, \vdash)$ is isomorphic to $(W \times(E \times \mathbb{Z}), \circ)$.

Proof. The proof of (i) immediately follows from Lemmas 4 and 6 . Show that the statement (ii) holds. Define a bijection $\Upsilon$ of $\operatorname{End}(E \times \mathbb{Z}, \dashv, \vdash)$ into $(W \times(E \times \mathbb{Z}), \circ)$ by

$$
\xi_{e, t} \Upsilon=(e, t) \text { for all } \xi_{e, t} \in \operatorname{End}(E \times \mathbb{Z}, \dashv, \vdash) .
$$

Let $\xi_{e, t}, \xi_{i, s} \in \operatorname{End}(E \times \mathbb{Z}, \dashv, \vdash)$ and $(a, n) \in E \times \mathbb{Z}$. We have the following four cases.

1) $e_{1}=t_{1}=1$. Then

$$
\begin{gathered}
(1, n) \xi_{e, t} \xi_{i, s}=\left(1, n t^{+}+t_{2}\right) \xi_{i, s}=\left(s_{1}, s_{2}+\left(n t^{+}+t_{2}\right) s^{+}\right)= \\
=\left(s_{1}, n t^{+} s^{+}+\left(t_{2} s^{+}+s_{2}\right)\right)=(1, n) \xi_{\left(s_{1},-s_{1}\right),\left(s_{1}, s_{2}+t_{2} s^{+}\right)} \\
(-1, n) \xi_{e, t} \xi_{i, s}=\left(1,(n-1) t^{+}+e_{2}\right) \xi_{i, s}=\left(s_{1}, s_{2}+\left((n-1) t^{+}+e_{2}\right) s^{+}\right)= \\
=\left(s_{1},(n-1) t^{+} s^{+}+\left(s_{2}-s^{+}\right)\right)=(-1, n) \xi_{\left(s_{1},-s_{1}\right),\left(s_{1}, s_{2}+t_{2} s^{+}\right)} .
\end{gathered}
$$

Thus, $\xi_{(1,-1),\left(1, t_{2}\right)} \xi_{i, s}=\xi_{\left(s_{1},-s_{1}\right),\left(s_{1}, s_{2}+t_{2} s^{+}\right)}$.

2) $e_{1}=1, t_{1}=-1$. Then

$$
\begin{gathered}
(1, n) \xi_{e, t} \xi_{i, s}=\left(-1, n t^{+}+t_{2}\right) \xi_{i, s}=\left(i_{1}, i_{2}+\left(n t^{+}+t_{2}-1\right) s^{+}\right)= \\
=\left(i_{1}, n t^{+} s^{+}+\left(t^{+} s^{+}-i_{1}\right)\right)=(1, n) \xi_{\left(s_{1},-s_{1}\right),\left(i_{1}, t^{+} s^{+}-i_{1}\right)}, \\
(-1, n) \xi_{e, t} \xi_{i, s}=\left(1,(n-1) t^{+}+e_{2}\right) \xi_{i, s}=\left(s_{1},\left((n-1) t^{+}+e_{2}\right) s^{+}+s_{2}\right)= \\
=\left(s_{1},(n-1) t^{+} s^{+}-s_{1}\right)=(-1, n) \xi_{\left(s_{1},-s_{1}\right),\left(i_{1}, t^{+} s^{+}-i_{1}\right)} .
\end{gathered}
$$

So, $\xi_{(1,-1),\left(-1, t_{2}\right)} \xi_{i, s}=\xi_{\left(s_{1},-s_{1}\right),\left(i_{1}, t^{+} s^{+}-i_{1}\right)}$.

3) $e_{1}=-1, t_{1}=1$. Then

$$
\begin{gathered}
(1, n) \xi_{e, t} \xi_{i, s}=\left(1, n t^{+}+t_{2}\right) \xi_{i, s}=\left(s_{1}, n t^{+} s^{+}+\left(t_{2} s^{+}+s_{2}\right)\right)=(1, n) \xi_{\left(i_{1},-i_{1}\right),\left(s_{1}, t_{2} s^{+}+s_{2}\right)} \\
(-1, n) \xi_{e, t} \xi_{i, s}=\left(-1,(n-1) t^{+}+e_{2}\right) \xi_{i, s}=\left(i_{1}, i_{2}+\left((n-1) t^{+}+e_{2}-1\right) s^{+}\right)= \\
=\left(i_{1},(n-1) t^{+} s^{+}-i_{1}\right)=(-1, n) \xi_{\left(i_{1},-i_{1}\right),\left(s_{1}, t_{2} s^{+}+s_{2}\right)} .
\end{gathered}
$$

Therefore, $\xi_{(-1,1),\left(1, t_{2}\right)} \xi_{i, s}=\xi_{\left(i_{1},-i_{1}\right),\left(s_{1}, t_{2} s^{+}+s_{2}\right)}$. 
4) $e_{1}=t_{1}=-1$. Then

$$
\begin{gathered}
\left.(1, n) \xi_{e, t} \xi_{i, s}=\left(-1, n t^{+}+t_{2}\right) \xi_{i, s}=\left(i_{1},\left(n t^{+}+t_{2}-1\right) s^{+}+i_{2}\right)\right)= \\
=\left(i_{1}, n t^{+} s^{+}+\left(t^{+} s^{+}-i_{1}\right)\right)=(1, n) \xi_{\left(i_{1},-i_{1}\right),\left(i_{1}, t^{+} s^{+}-i_{1}\right)}, \\
(-1, n) \xi_{e, t} \xi_{i, s}=\left(-1,(n-1) t^{+}+e_{2}\right) \xi_{i, s}=\left(i_{1}, i_{2}+\left((n-1) t^{+}+e_{2}-1\right) s^{+}\right)= \\
=\left(i_{1},(n-1) t^{+} s^{+}-i_{1}\right)=(-1, n) \xi_{\left(i_{1},-i_{1}\right),\left(i_{1}, t^{+} s^{+-} i_{1}\right)} .
\end{gathered}
$$

Thus, $\xi_{(-1,1),\left(-1, t_{2}\right)} \xi_{i, s}=\xi_{\left(i_{1},-i_{1}\right),\left(i_{1}, t^{+} s^{+}-i_{1}\right)}$.

Finally, using equalities from cases 1)-4), we obtain

$$
\left(\xi_{e, t} \xi_{i, s}\right) \Upsilon= \begin{cases}\left(\left(s_{1},-s_{1}\right),\left(s_{1}, t_{2} s^{+}+s_{2}\right)\right), & \text { if } e_{1}=t_{1}=1 \\ \left(\left(s_{1},-s_{1}\right),\left(i_{1}, t^{+} s^{+}-i_{1}\right)\right), & \text { if } e_{1}=1, t_{1}=-1, \\ \left(\left(i_{1},-i_{1}\right),\left(s_{1}, t_{2} s^{+}+s_{2}\right)\right), & \text { if } e_{1}=-1, t_{1}=1 \\ \left(\left(i_{1},-i_{1}\right),\left(i_{1}, t^{+} s^{+}-i_{1}\right)\right), & \text { if } e_{1}=t_{1}=-1\end{cases}
$$

for all $\xi_{e, t}, \xi_{i, s} \in \operatorname{End}(E \times \mathbb{Z}, \dashv, \vdash)$.

On the other hand,

$$
\xi_{e, t} \Upsilon \circ \xi_{i, s} \Upsilon= \begin{cases}\left((1,-1),\left(1, t_{2}\right)\right) \circ(i, s)=\left(\left(s_{1},-s_{1}\right),\left(s_{1}, t_{2} s^{+}+s_{2}\right)\right), & \text { if } e_{1}=t_{1}=1, \\ \left((1,-1),\left(-1, t_{2}\right)\right) \circ(i, s)=\left(\left(s_{1},-s_{1}\right),\left(i_{1}, t^{+} s^{+}-i_{1}\right)\right), & \text { if } e_{1}=1, t_{1}=-1, \\ \left((-1,1),\left(1, t_{2}\right)\right) \circ(i, s)=\left(\left(i_{1},-i_{1}\right),\left(s_{1}, t_{2} s^{+}+s_{2}\right)\right), & \text { if } e_{1}=-1, t_{1}=1, \\ \left((-1,1),\left(-1, t_{2}\right)\right) \circ(i, s)=\left(\left(i_{1},-i_{1}\right),\left(i_{1}, t^{+} s^{+}-i_{1}\right)\right), & \text { if } e_{1}=t_{1}=-1,\end{cases}
$$

which completes the proof of this theorem.

Observe that the automorphism group of the free abelian digroup $(E \times \mathbb{Z}, \dashv, \vdash)$ is twoelement, that is, $\operatorname{Aut}(E \times \mathbb{Z}, \dashv, \vdash)=\left\{\xi_{(-1,1),(1,0)}, \xi_{(1,-1),(-1,0)}\right\}$.

\section{REFERENCES}

1. Loday J.-L., Dialgebras, in: Dialgebras and related operads, Lect. Notes Math., 1763 (2001) SpringerVerlag, Berlin, 7-66.

2. Liu K., Transformation digroups, preprint, available at http://arxiv.org/abs/math/0409265.

3. Kinyon M.K., Leibniz algebras, Lie racks, and digroups, J. Lie Theory, 17 (2007), №1, 99-114.

4. Phillips J.D., A short basis for the variety of digroups, Semigroup Forum, 70 (2005), 466-470.

5. Crompton C., Scalici L., The structure of digroups, American Journal of Undergraduate research, 5 (2006), №2, 21-27.

6. Felipe R., Generalized Loday algebras and digroups, Comunicaciones del CIMAT, №I-04-01/21-01-2004.

7. Liu K., The generalizations of groups, Research Monographs in Math., V.1, 153 Publishing: Burnaby, 2004.

8. Zhuchok A.V., Dimonoids, Algebra and Logic, 50 (2011), №4, 323-340.

9. Zhuchok A.V., Free commutative dimonoids, Algebra and Discrete Mathematics, 9 (2010), №1, 109-119.

10. Zhuchok Yu.V., Representations of ordered dimonoids by binary relations, Asian-European J. Math., 7 (2014), 1-13.

Kyiv National Taras Schevchenko University Faculty of Mechanics and Mathematics zhuchok_y@mail.ru 